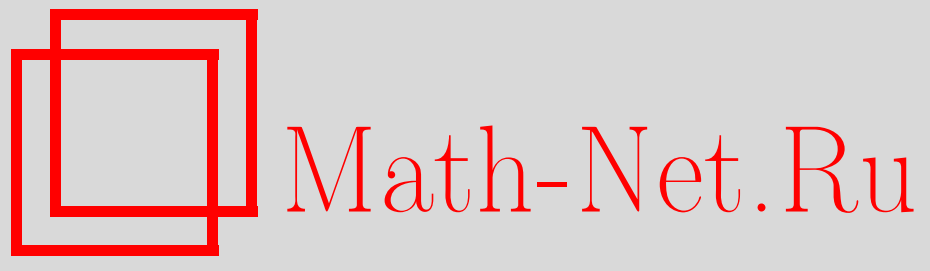

N.

Watanabe,

Note on complexity of quantum transmission processes, Вестн. Сам. гос. техн. ун-та. Сер. Физ.-мат. науки, 2013, выпуск 1(), 305-314

DOI: https://doi.org/10.14498/vsgtu1127

Использование Общероссийского математического портала Math-Net.Ru подразумевает, что вы прочитали и согласны с пользовательским соглашением

http://www . mathnet.ru/rus/agreement

Параметры загрузки:

IP: 54.80 .97 .219

26 апреля 2023 г., 15:06:54

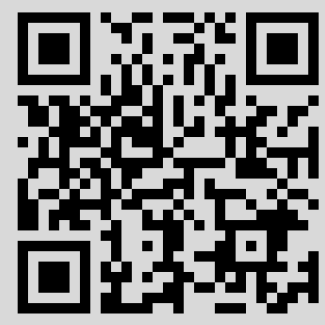


UDC 004.38:530.145; MSC: 81P99; 81V80, 94A50

\title{
NOTE ON COMPLEXITY OF QUANTUM TRANSMISSION PROCESSES
}

\author{
N. Watanabe \\ Science University of Tokyo, Noda City, Chiba 278-8510, Japan. \\ E-mail: watanabe@is.noda.tus.ac.jp
}

In 1989, Ohya propose a new concept, so-called Information Dynamics (ID), to investigate complex systems according to two kinds of view points. One is the dynamics of state change and another is measure of complexity. In ID, two complexities $C^{S}$ and $T^{S}$ are introduced. $C^{S}$ is a measure for complexity of system itself, and $T^{S}$ is a measure for dynamical change of states, which is called a transmitted complexity. An example of these complexities of ID is entropy for information transmission processes. The study of complexity is strongly related to the study of entropy theory for classical and quantum systems. The quantum entropy was introduced by von Neumann around 1932, which describes the amount of information of the quantum state itself. It was extended by Ohya for $C^{*}$-systems before CNT entropy. The quantum relative entropy was first defined by Umegaki for $\sigma$-finite von Neumann algebras, which was extended by Araki and Uhlmann for general von Neumann algebras and *-algebras, respectively. By introducing a new notion, the so-called compound state, in 1983 Ohya succeeded to formulate the mutual entropy in a complete quantum mechanical system (i.e., input state, output state and channel are all quantum mechanical) describing the amount of information correctly transmitted through the quantum channel. In this paper, we briefly review the entropic complexities for classical and quantum systems. We introduce some complexities by means of entropy functionals in order to treat the transmission processes consistently. We apply the general frames of quantum communication to the Gaussian communication processes. Finally, we discuss about a construction of compound states including quantum correlations.

Key words: quantum communication channel, von Neumann entropy, $\mathcal{S}$-mixing entropy, Ohya mutual entropy, $C^{*}$-system.

\section{Introduction}

In [1], Ohya introduced Information Dynamics (ID) synthesizing dynamics of state change and complexity of state. Based on ID, one can study various problems of physics and other fields. Channel and two complexities are key concepts of ID.

Let us briefly review ID for quantum communication processes.

Let $\mathcal{H}_{k}(k=1,2)$ be complex separable Hilbert spaces. We denote the set of all bounded linear operators on $\mathcal{H}_{k}$ by $\mathbf{B}\left(\mathcal{H}_{k}\right)(k=1,2)$ and we express the set of all density operators on $\mathcal{H}_{k}$ by $\mathfrak{S}\left(\mathcal{H}_{k}\right)(k=1,2)$. Let $\left(\mathbf{B}\left(\mathcal{H}_{k}\right), \mathfrak{S}\left(\mathcal{H}_{k}\right)\right)(k=1,2)$ be input $(k=1)$ and output $(k=2)$ quantum systems, respectively.

\subsection{Quantum Channels}

A mapping from $\mathfrak{S}\left(\mathcal{H}_{1}\right)$ to $\mathfrak{S}\left(\mathcal{H}_{2}\right)$ is called a quantum channel $\Lambda^{*}$.

(1) $\Lambda^{*}$ is called a linear channel if $\Lambda^{*}$ satisfies the affine property such as $\Lambda^{*}\left(\sum_{k} \lambda_{k} \rho_{k}\right)=\sum_{k} \lambda_{k} \Lambda^{*}\left(\rho_{k}\right)$ for any $\rho_{k} \in \mathfrak{S}\left(\mathcal{H}_{1}\right)$ and any nonnegative number $\lambda_{k} \in[0,1]$ with $\sum_{k} \lambda_{k}=1$.

For the quantum channel $\Lambda^{*}$, the dual map $\Lambda$ of $\Lambda^{*}$ is defined by

$$
\operatorname{tr} \Lambda^{*}(\rho) B=\operatorname{tr} \rho \Lambda(B), \quad \forall \rho \in \mathfrak{S}\left(\mathcal{H}_{1}\right), \forall B \in \mathbf{B}\left(\mathcal{H}_{2}\right) .
$$

Noboru Watanabe, Professor, Dept. of Information Sciences. 
(2) $\Lambda^{*}$ is called a completely positive (CP) channel if $\Lambda^{*}$ is linear channel and its dual map $\Lambda: \mathbf{B}\left(\mathcal{H}_{2}\right) \rightarrow \mathbf{B}\left(\mathcal{H}_{1}\right)$ of $\Lambda^{*}$ holds

$$
\left\langle x, \sum_{i, j=1}^{n} A_{i}^{*} \Lambda\left(\bar{A}_{i}^{*} \bar{A}_{j}\right) A_{j} x\right\rangle \geqslant 0 \quad\left(\forall x \in \mathcal{H}_{1}\right)
$$

for any $n \in \mathbf{N}$, any $\left\{\bar{A}_{i}\right\} \subset \mathbf{B}\left(\mathcal{H}_{2}\right)$ and any $\left\{A_{i}\right\} \subset \mathbf{B}\left(\mathcal{H}_{1}\right)$.

One can describe almost all physical transform of states by using the CP channel [2-5].

\subsection{Quantum Communication Channel}

Here we explain the quantum communication channels as an example of the quantum channels.

In order to consider influence of the environment such as noise and loss, we suppose $\mathcal{K}_{1}$ and $\mathcal{K}_{2}$ to be complex separable Hilbert spaces of noise and loss systems, respectively. Quantum channel of quantum communication process with noise and loss was discussed by $[2,6]$.

\subsection{Noisy quantum channel and Generalized Beam Splitter}

For an input state $\rho$ in $\mathfrak{S}\left(\mathcal{H}_{1}\right)$ and a noise state $\xi \in \mathfrak{S}\left(\mathcal{K}_{1}\right)$, Ohya and NW defined in [6] a generalized beam splitting $\Pi^{*}$ by

$$
\Pi^{*}(\rho \otimes \xi) \equiv V(\rho \otimes \xi) V^{*}
$$

where $V$ is a linear mapping from $\mathcal{H}_{1} \otimes \mathcal{K}_{1}$ to $\mathcal{H}_{2} \otimes \mathcal{K}_{2}$ given by for the $n_{1}, m_{1}$, $j,\left(n_{1}+m_{1}-j\right)$ photon number state vectors $\left|n_{1}\right\rangle \in \mathcal{H}_{1},\left|m_{1}\right\rangle \in \mathcal{K}_{1},|j\rangle \in \mathcal{H}_{2}$, $\left|n_{1}+m_{1}-j\right\rangle \in \mathcal{K}_{2}$

$$
V\left(\left|n_{1}\right\rangle \otimes\left|m_{1}\right\rangle\right)=\sum_{j}^{n_{1}+m_{1}} C_{j}^{n_{1}, m_{1}}|j\rangle \otimes\left|n_{1}+m_{1}-j\right\rangle
$$

and

$$
\begin{aligned}
C_{j}^{n_{1}, m_{1}}= & \sum_{\substack{r=L\\
}}^{K}(-1)^{n_{1}+j-r} \frac{\sqrt{n_{1} ! m_{1} ! j !\left(n_{1}+m_{1}-j\right) !}}{r !\left(n_{1}-j\right) !(j-r) !\left(m_{1}-j+r\right) !} \\
& \times \alpha^{m_{1}-j+2 r}(-\bar{\beta})^{n_{1}+j-2 r}
\end{aligned}
$$

$\alpha$ and $\beta$ are complex numbers satisfying $|\alpha|^{2}+|\beta|^{2}=1 . K$ and $L$ are constants given by $K=\min \left\{n_{1}, j\right\}, L=\max \left\{m_{1}-j, 0\right\}$. For the coherent input state $\rho=|\theta\rangle\langle\theta|\otimes| \kappa\rangle\langle\kappa| \in \mathfrak{S}\left(\mathcal{H}_{1} \otimes \mathcal{K}_{1}\right)$, the output state of $\Pi^{*}$ is obtained by

$$
\begin{aligned}
\Pi^{*}(|\theta\rangle\langle\theta|\otimes| \kappa\rangle\langle\kappa|)= & |\alpha \theta+\beta \kappa\rangle\langle\alpha \theta+\beta \kappa| \\
& \otimes|-\bar{\beta} \theta+\bar{\alpha} \kappa\rangle\langle-\bar{\beta} \theta+\bar{\alpha} \kappa| .
\end{aligned}
$$

By using $\Pi^{*}$, Ohya and NW introduced in [6] the noisy quantum channel $\Lambda^{*}$ with a fixed noise state $\xi \in \mathfrak{S}\left(\mathcal{K}_{1}\right)$ defined by

$$
\Lambda^{*}(\rho) \equiv \operatorname{tr}_{\mathcal{K}_{2}} \Pi^{*}(\rho \otimes \xi)=\operatorname{tr}_{\mathcal{K}_{2}} V(\rho \otimes \xi) V^{*} .
$$


The generalized beam splitting $\Pi^{*}$ with the vacuum noise state $\xi_{0}=|0\rangle\langle 0|$ is called the beam splitter $\Pi_{0}^{*}$ given by

$$
\Pi_{0}^{*}\left(|\theta\rangle\langle\theta| \otimes \xi_{0}\right)=|\alpha \theta\rangle\langle\alpha \theta|\otimes|-\bar{\beta} \theta\rangle\langle-\bar{\beta} \theta|
$$

for the coherent input state $\rho \otimes \xi_{0}=|\theta\rangle\langle\theta|\otimes| 0\rangle\langle 0| \in \mathfrak{S}\left(\mathcal{H}_{1} \otimes \mathcal{K}_{1}\right)$. The beam splitter $\Pi_{0}^{*}$ was described by means of the lifting $\mathcal{E}_{0}^{*}$ from $\mathfrak{S}(\mathcal{H})$ to $\mathfrak{S}(\mathcal{H} \otimes \mathcal{K})$ in the sense of Accardi and Ohya [7] as follows

$$
\mathcal{E}_{0}^{*}(|\theta\rangle\langle\theta|)=|\alpha \theta\rangle\langle\alpha \theta|\otimes| \beta \theta\rangle\langle\beta \theta| \text {. }
$$

Based on the liftings, the beam splitting was studied by Accardi-Ohya and Fichtner-Freudenberg-Libsher [8]. Moreover, the noisy quantum channel $\Lambda_{0}^{*}$ with the vacuum noise state $\xi_{0}=|0\rangle\langle 0|$ is called the attenuation channel given by Ohya [2] as

$$
\Lambda_{0}^{*}(\rho) \equiv \operatorname{tr}_{\mathcal{K}_{2}} \Pi_{0}^{*}\left(\rho \otimes \xi_{0}\right)=\operatorname{tr}_{\mathcal{K}_{2}} V_{0}(\rho \otimes|0\rangle\langle 0|) V_{0}^{*},
$$

which plays an important role for investigating the quantum communication processes.

\section{Complexities}

Two kind of complexities $C^{\mathcal{S}}(\rho), T^{\mathcal{S}}\left(\rho ; \Lambda^{*}\right)$ are used in ID. $C^{\mathcal{S}}(\rho)$ is a complexity of a state $\rho$ measured from a subset $\mathcal{S}$ and $T^{\mathcal{S}}\left(\rho ; \Lambda^{*}\right)$ is a transmitted complexity according to the state change from $\rho$ to $\Lambda^{*} \rho$. These complexities should fulfill the following conditions: Let $\mathcal{S}, \overline{\mathcal{S}}, \mathcal{S}_{t}$ be subsets of $\mathfrak{S}\left(\mathcal{H}_{1}\right), \mathfrak{S}\left(\mathcal{H}_{2}\right)$, $\mathfrak{S}\left(\mathcal{H}_{1} \otimes \mathcal{H}_{2}\right)$, respectively.

(1) For any $\rho \in \mathcal{S}, C^{\mathcal{S}}(\rho)$ and $T^{\mathcal{S}}\left(\rho ; \Lambda^{*}\right)$ are nonnegative $\left(C^{\mathcal{S}}(\rho) \geqslant 0\right.$, $\left.T^{\mathcal{S}}\left(\rho ; \Lambda^{*}\right) \geqslant 0\right)$.

(2) For a bijection $j$ from ex $\mathfrak{S}\left(\mathcal{H}_{1}\right)$ to ex $\mathfrak{S}\left(\mathcal{H}_{1}\right)$,

$$
C^{\mathcal{S}}(\rho)=C^{\mathcal{S}}(j(\rho))
$$

is hold, where ex $\mathfrak{S}\left(\mathcal{H}_{1}\right)$ is the set of extremal point of $\mathfrak{S}\left(\mathcal{H}_{1}\right)$.

(3) For $\rho \otimes \sigma \in \mathfrak{S}\left(\mathcal{H}_{1} \otimes \mathcal{H}_{2}\right), \rho \in \mathfrak{S}\left(\mathcal{H}_{1}\right), \sigma \in \mathfrak{S}\left(\mathcal{H}_{2}\right)$,

$$
C^{\mathcal{S}_{t}}(\rho \otimes \sigma)=C^{\mathcal{S}}(\rho)+C^{\overline{\mathcal{S}}}(\sigma)
$$

It means that the complexity of the state $\rho \otimes \sigma$ of totally independent systems are given by the sum of the complexities of the states $\rho$ and $\sigma$.

(4) $C^{\mathcal{S}}(\rho)$ and $T^{\mathcal{S}}\left(\rho ; \Lambda^{*}\right)$ satisfy the following inequality $0 \leqslant T^{\mathcal{S}}\left(\rho ; \Lambda^{*}\right) \leqslant$ $C^{\mathcal{S}}(\rho)$.

(5) If the channel $\Lambda^{*}$ is given by the identity map $i d$, then $T^{\mathcal{S}}(\rho ; i d)=C^{\mathcal{S}}(\rho)$ is hold.

One of the example of the above complexities are the Shannon entropy $S(p)$ for $C^{\mathcal{S}}(p)$ and classical mutual entropy $I\left(p ; \Lambda^{*}\right)$ for $T^{\mathcal{S}}\left(p ; \Lambda^{*}\right)$. Let us consider these complexities for quantum systems.

\subsection{Example of Complexity $C^{\mathcal{S}}(\rho)$}

\subsection{1. von Neumann Entropy and $\mathcal{S}$-mixing entropy}

One of the example of the complexity $C^{\mathcal{S}}(\rho)$ of ID in quantum system is the von Neumann entropy $S(\rho)[9]$ described by

$$
C^{\mathcal{S}}(\rho) \Leftrightarrow S(\rho)=-\operatorname{tr} \rho \log \rho
$$


for any density operators $\rho \in \mathfrak{S}\left(\mathcal{H}_{1}\right)$, which satisfies the above conditions (1), (2), (3).

Let $(\mathcal{A}, \mathfrak{S}(\mathcal{A}), \alpha(G))$ be a $\mathrm{C}^{*}$-dynamical system and $\mathcal{S}$ be a weak ${ }^{*}$ compact and convex subset of $\mathfrak{S}(\mathcal{A})$. For example, $\mathcal{S}$ is given by $\mathfrak{S}(\mathcal{A})$ (the set of all states on $\mathcal{A}), I(\alpha)$ (the set of all invariant states for $\alpha$ ), $K(\alpha)$ (the set of all KMS states), and so on. Every state $\varphi \in \mathcal{S}$ has a maximal measure $\mu$ pseudosupported on ex $\mathcal{S}$ such that

$$
\varphi=\int_{\mathcal{S}} \omega d \mu
$$

where ex $\mathcal{S}$ is the set of all extreme points of $\mathcal{S}$. The measure $\mu$ giving the above decomposition is not unique unless $\mathcal{S}$ is a Choquet simplex. We denote the set of all such measures by $M_{\varphi}(\mathcal{S})$, and define

$$
\begin{gathered}
D_{\varphi}(\mathcal{S})=\left\{M_{\varphi}(\mathcal{S}) ; \quad \exists \mu_{k} \subset \mathbb{R}^{+} \text {and }\left\{\varphi_{k}\right\} \subset \operatorname{ex} S\right. \\
\text { s.t. } \left.\quad \sum_{k} \mu_{k}=1, \quad \mu=\sum_{k} \mu_{k} \delta\left(\varphi_{k}\right)\right\}
\end{gathered}
$$

where $\delta(\varphi)$ is the Dirac measure concentrated on an initial state $\varphi$. For a measure $\mu \in D_{\varphi}(\mathcal{S})$, we put

$$
H(\mu)=-\sum_{k} \mu_{k} \log \mu_{k}
$$

The $\mathrm{C}^{*}$-entropy of a state $\varphi \in \mathcal{S}$ with respect to $\mathcal{S}$ ( $\mathcal{S}$-mixing entropy) is defined by

$$
C^{\mathcal{S}}(\varphi) \Leftrightarrow S^{\mathcal{S}}(\varphi)=\left\{\begin{array}{lr}
\inf \left\{H(\mu) ; \quad \mu \in D_{\varphi}(\mathcal{S})\right\} \\
+\infty & \text { if } D_{\varphi}(\mathcal{S})=\varnothing .
\end{array}\right.
$$

It describes the amount of information of the state $\varphi$ measured from the subsystem $\mathcal{S}$. We denote $S^{\mathfrak{S}(\mathcal{A})}(\varphi)$ by $S(\varphi)$ if $\mathcal{S}=\mathfrak{S}(\mathcal{A})$. It is an extension of von Neumann's entropy.

This entropy (mixing $\mathcal{S}$-entropy) of a general state $\varphi$ satisfies the following properties [10].

Theorem 2.1. When $\mathcal{A}=B(\mathcal{H})$ and $\alpha_{t}=A d\left(U_{t}\right)$ (i.e., $\alpha_{t}(A)=U_{t}^{*} A U_{t}$ for any $A \in \mathcal{A})$ with a unitary operator $U_{t}$, for any state $\varphi$ given by $\varphi(\cdot)=\operatorname{tr} \rho$. with a density operator $\rho$, the following facts hold:

(1) $S(\varphi)=-\operatorname{tr} \rho \log \rho$.

(2) If $\varphi$ is an $\alpha$-invariant faithful state and every eigenvalue of $\rho$ is nondegenerate, then $S^{I(\alpha)}(\varphi)=S(\varphi)$, where $I(\alpha)$ is the set of all $\alpha$-invariant faithful states.

(3) If $\varphi \in K(\alpha)$, then $S^{K(\alpha)}(\varphi)=0$, where $K(\alpha)$ is the set of all KMS states.

TheOREM 2.2. For any $\varphi \in K(\alpha)$, we have

(1) $S^{K(\alpha)}(\varphi) \leqslant S^{I(\alpha)}(\varphi)$.

(2) $S^{K(\alpha)}(\varphi) \leqslant S(\varphi)$.

\subsection{Example of Transmitted Complexity $T^{\mathcal{S}}\left(\rho ; \Lambda^{*}\right)$}

The classical mutual entropy $I\left(p ; \Lambda^{*}\right)$ defined by using the joint probability distribution between the input state and the output state is an example of the transmitted complexity $T^{\mathcal{S}}\left(p ; \Lambda^{*}\right)$ of ID. In general, there does not exit the joint 
states in the quantum system [11]. We need to introduce the compound state in quantum system instead of the joint probability distribution in classical system.

\subsection{Compound state} ditions:

The quantum mutual entropy $I\left(\rho, \Lambda^{*}\right)$ should satisfy the following three con-

1) If the channel is given by the identity channel $i d$, then $I(\rho ; i d)=S(\rho)$ (von Neumann entropy) is hold.

2) If the system is classical, then the quantum mutual equals to the classical mutual entropy.

3) The quantum mutual entropy should satisfy the Shannon's type inequalities:

$$
0 \leqslant I\left(\rho, \Lambda^{*}\right) \leqslant S(\rho) .
$$

Ohya introduced two compound states $\sigma_{0}$ and $\sigma_{E} \cdot \sigma_{0}$ is the trivial compound state given by

$$
\sigma_{0}=\rho \otimes \Lambda^{*} \rho .
$$

$\sigma_{E}$ is the compound state representing a certain correlation between the input state and the output state given by

$$
\sigma_{E}=\sum_{n} \lambda_{n} E_{n} \otimes \Lambda^{*} E_{n}
$$

associated with the Schatten-von Neumann (one dimensional spectral) decomposition [12] $\rho=\sum_{n} \lambda_{n} E_{n}$ of the input state $\rho$.

\subsection{Ohya Mutual Entropy for density operator}

An example of the transmitted complexity $T^{\mathcal{S}}\left(\rho ; \Lambda^{*}\right)$ of ID in quantum system is the Ohya mutual entropy with respect to the initial state $\rho$ and the quantum channel $\Lambda^{*}$ defined by

$$
T^{\mathcal{S}}\left(\rho ; \Lambda^{*}\right) \Leftrightarrow I\left(\rho ; \Lambda^{*}\right) \equiv \sup \left\{\sum_{n} S\left(\sigma_{E}, \sigma_{0}\right), \rho=\sum_{n} \lambda_{n} E_{n}\right\},
$$

where $S(\cdot, \cdot)$ is the Umegaki's relative entropy [13] denoted by

$$
S(\rho, \sigma) \equiv\left\{\begin{array}{cc}
\operatorname{tr} \rho(\log \rho-\log \sigma) & (\text { when } \overline{\operatorname{ran} \rho} \subset \overline{\operatorname{ran} \sigma}) \\
\infty & \text { (otherwise) }
\end{array}\right.
$$

which was extended to more general quantum systems by Araki and Uhlmann $[1,3,4,14,16]$. The Ohya mutual entropy holds the above conditions (1), (4), (5) such as

$$
\begin{gathered}
0 \leqslant I\left(\rho, \Lambda^{*}\right) \leqslant S(\rho), \\
I(\rho, i d)=S(\rho) .
\end{gathered}
$$

The capacity means the ability of the information transmission of the channel, which is used as a measure for construction of channels. The quantum capacity is formulated by taking the supremum of the Ohya mutual entropy with respect to a certain subset of the initial state space. The quantum capacity of quantum channel was studied in [17-20]. 
TheOREM 2.3. Let $\Phi_{E}$ be a compound state w.r.t. the initial state $\rho$, the quantum CP channel $\Lambda^{*}$ and aSchatten decomposition of $\rho=\sum_{k} \lambda_{k} E_{k}$ defined by

$$
\Phi_{E}=\sum_{n}\left(I \otimes V_{n}\right)\left[\sum_{k} \sqrt{\lambda_{k}}\left|x_{k}\right\rangle \otimes\left|x_{k}\right\rangle\right]\left[\sum_{k^{\prime}} \sqrt{\lambda_{k^{\prime}}}\left\langle x_{k^{\prime}}\right| \otimes\left\langle x_{k^{\prime}}\right|\right]\left(I \otimes V_{n}^{*}\right)
$$

under the condition

$$
\sum_{n}\left(I \otimes V_{n}^{*}\right)\left(I \otimes V_{n}\right)=I \otimes I
$$

and $\Lambda^{*}$ is given by $\Lambda^{*}(\rho)=\sum_{n} V_{n} \rho V_{n}^{*}$. By defining the compound state $\Phi_{E}$, one can obtain the following theorem.

TheOREM 2.4. For the compound state $\Phi_{E}$ given above, one can obtain two marginal states as follows

$$
\begin{array}{r}
\operatorname{tr}_{\mathcal{H}_{2}} \Phi_{E}=S(\rho), \\
\operatorname{tr}_{\mathcal{H}_{1}} \Phi_{E}=S\left(\Lambda^{*} \rho\right) .
\end{array}
$$

The upper bound of the relative entropy $S\left(\Phi_{E}, \rho \otimes \Lambda^{*} \rho\right)$ is obtained as follows:

$$
S\left(\Phi_{E}, \rho \otimes \Lambda^{*} \rho\right) \leqslant 2 S(\rho) .
$$

Let $\Psi_{E}$ be a compoundstate defined by

$$
\Psi_{E, \mu}=\mu \sigma_{E}+(1-\mu) \Phi_{E} \quad(\mu \in[0,1]) .
$$

We have the following theorem.

TheOREM 2.5. For the compound state $\Psi_{E, \mu}$ w.r.t. $\mu \in[0,1]$ given above, one can obtain two marginal states as follows

$$
\begin{array}{r}
\operatorname{tr}_{\mathcal{H}_{2}} \Psi_{E, \mu}=S(\rho), \\
\operatorname{tr}_{\mathcal{H}_{1}} \Psi_{E, \mu}=S\left(\Lambda^{*} \rho\right) .
\end{array}
$$

The upper bound of the relative entropy $S\left(\Psi_{E, \mu}, \rho \otimes \Lambda^{*} \rho\right)$ is obtained as follows:

$$
S\left(\Psi_{E, \mu}, \rho \otimes \Lambda^{*} \rho\right) \leqslant(2-\mu) S(\rho) \quad(\mu \in[0,1]) .
$$

\subsection{Ohya Mutual Entropy for general $\mathrm{C}^{*}$-system}

Let $(\mathcal{A}, \mathfrak{S}(\mathcal{A}), \alpha(G))$ be a unital $C^{*}$-system and $\mathcal{S}$ be a weak* compact convex subset of $\mathfrak{S}(\mathcal{A})$. For an initial state $\varphi \in \mathcal{S}$ and a channel $\Lambda^{*}: \mathfrak{S}(\mathcal{A}) \rightarrow \mathfrak{S}(\mathcal{B})$, two compound states $[3,10]$ are defined by

$$
\begin{gathered}
\Phi_{\mu}^{\mathcal{S}}=\int_{\mathcal{S}} \omega \otimes \Lambda^{*} \omega d \mu \\
\Phi_{0}=\varphi \otimes \Lambda^{*} \varphi
\end{gathered}
$$


The compound state $\Phi_{\mu}^{\mathcal{S}}$ expresses the correlation between the input state $\varphi$ and the output state $\Lambda^{*} \varphi$. The mutual entropy with respect to $\mathcal{S}$ and $\mu$ is given by

$$
I_{\mu}^{\mathcal{S}}\left(\varphi ; \Lambda^{*}\right)=S\left(\Phi_{\mu}^{\mathcal{S}}, \Phi_{0}\right)
$$

and the mutual entropy with respect to $\mathcal{S}$ is defined by Ohya $[3,10]$ as

$$
T^{\mathcal{S}}\left(\varphi ; \Lambda^{*}\right) \Leftrightarrow I^{\mathcal{S}}\left(\varphi ; \Lambda^{*}\right)=\sup \left\{I_{\mu}^{\mathcal{S}}\left(\varphi ; \Lambda^{*}\right) ; \mu \in M_{\varphi}(\mathcal{S})\right\}
$$

\subsection{Other Mutual Entropy Type Measures}

Recently, several mutual entropy type measures were proposed by Shor [21] and Bennet et al [22,23], which defined by using the entropy exchange [24] given by

$$
S_{e}\left(\rho, \Lambda^{*}\right)=-\operatorname{tr} W \log W,
$$

where $W$ is a matrix $W=\left(W_{i j}\right)_{i, j}$ with the elements

$$
W_{i j} \equiv \operatorname{tr} A_{i}^{*} \rho A_{j}
$$

obtained by means of the input state $\rho$ and the CP channel $\Lambda^{*}$ described by a Stinespring-Sudarshan-Kraus form

$$
\Lambda^{*}(\cdot) \equiv \sum_{j} A_{j}^{*} \cdot A_{j}
$$

Based on the entropy exchange, the coherent entropy $I_{C}\left(\rho ; \Lambda^{*}\right)[15]$ and the Lindblad-Nielson entropy $I_{L}\left(\rho ; \Lambda^{*}\right)[23]$ were defined by

$$
\begin{gathered}
I_{C}\left(\rho ; \Lambda^{*}\right) \equiv S\left(\Lambda^{*} \rho\right)-S_{e}\left(\rho, \Lambda^{*}\right), \\
I_{L}\left(\rho ; \Lambda^{*}\right) \equiv S(\rho)+S\left(\Lambda^{*} \rho\right)-S_{e}\left(\rho, \Lambda^{*}\right) .
\end{gathered}
$$

\subsection{Comparison among these quantum mutual entropy type measures}

In this section, we compare with these mutual types measures.

By comparing these mutual entropies for quantum information communication processes, we have the following theorem [25]:

Theorem 2.6. Let $\left\{A_{j}\right\}$ be a projection valued measure with dim $A_{j}=1$. For arbitrary state $\rho$ and the quantum channel $\Lambda^{*}(\cdot) \equiv \sum_{j} A_{j} \cdot A_{j}^{*}$, one has

(1) $0 \leqslant I\left(\rho ; \Lambda^{*}\right) \leqslant \min \left\{S(\rho), S\left(\Lambda^{*} \rho\right)\right\}$ (Ohya mutual entropy),

(2) $I_{C}\left(\rho ; \Lambda^{*}\right)=0$ (coherent entropy),

(3) $I_{L}\left(\rho ; \Lambda^{*}\right)=S(\rho)$ (Lindblad entropy).

For the attenuation channel $\Lambda_{0}^{*}$, one can obtain the following theorems [25]:

Lemma 2.1. For the attenuation channel $\Lambda_{0}^{*}$ and the input state

$$
\rho=\lambda|0\rangle\langle 0|+(1-\lambda)| \theta\rangle\langle\theta|
$$

there exists a unitary operator $U$ such that

$$
U W U^{*}=\lambda|0\rangle\langle 0|+(1-\lambda)|-\bar{\beta} \theta\rangle\langle-\bar{\beta} \theta| .
$$


Theorem 2.7. For the attenuation channel $\Lambda_{0}^{*}$ and the input state

$$
\rho=\lambda|0\rangle\langle 0|+(1-\lambda)| \theta\rangle\langle\theta|,
$$

the entropy exchange is obtained by

$$
S_{e}\left(\rho, \Lambda_{0}^{*}\right)=-\operatorname{tr} W \log W=-\sum_{j=0}^{1} \mu_{j} \log \mu_{j}
$$

where

$$
\left.\mu_{j}=\frac{1}{2}\left\{1+(-1)^{j} \sqrt{1-4 \lambda(1-\lambda)\left(1-\exp \left(-|\beta|^{2}|\theta|^{2}\right)\right.}\right)\right\} \quad(j=0,1) .
$$

THEOREM 2.8. For any state $\rho=\sum_{n} \lambda_{n}|n\rangle\langle n|$ and the attenuation channel $\Lambda_{0}^{*}$ with $|\alpha|^{2}=|\beta|^{2}=\frac{1}{2}$, one has

(1) $0 \leqslant I\left(\rho ; \Lambda_{0}^{*}\right) \leqslant \min \left\{S(\rho), S\left(\Lambda_{0}^{*} \rho\right)\right\}$ (Ohya mutual entropy),

(2) $I_{C}\left(\rho ; \Lambda_{0}^{*}\right)=0$ (coherent entropy),

(3) $I_{L}\left(\rho ; \Lambda_{0}^{*}\right)=S(\rho)$ (Lindblad entropy).

TheOrem 2.9. For the attenuation channel $\Lambda_{0}^{*}$ and the input state

$$
\rho=\lambda|0\rangle\langle 0|+(1-\lambda)| \theta\rangle\langle\theta|
$$

we have

(1) $0 \leqslant I\left(\rho ; \Lambda_{0}^{*}\right) \leqslant \min \left\{S(\rho), S\left(\Lambda_{0}^{*} \rho\right)\right\}$ (Ohya mutual entropy),

(2) $-S(\rho) \leqslant I_{C}\left(\rho ; \Lambda_{0}^{*}\right) \leqslant S(\rho)$ (coherent entropy),

(3) $0 \leqslant I_{L}\left(\rho ; \Lambda_{0}^{*}\right) \leqslant 2 S(\rho)$ (Lindblad entropy).

It shows that the coherent entropy holds $I_{C}\left(\rho ; \Lambda_{0}^{*}\right)<0$ for $|\alpha|^{2}<|\beta|^{2}$ and the Lindblad entropy satisfies $I_{L}\left(\rho ; \Lambda_{0}^{*}\right) \geqslant S(\rho)$ for $|\alpha|^{2}>|\beta|^{2}$. From the above theorems, we can conclude that the transmitted complexity in quantum system is the Ohya mutual entropy and it is most fitting measure for studying the efficiency of information transmission in quantum communication processes. It means that Ohya mutual entropy can be considered as the transmitted complexity for quantum communication processes.

Theorem 2.10. For the attenuation channel $\Lambda_{0}^{*}$ and the input state

$$
\rho=\lambda|0\rangle\langle 0|+(1-\lambda)| \theta\rangle\langle\theta|,
$$

if $\lambda=\frac{1}{2}$ and $\beta=\sqrt{\frac{2}{3}}$, then there exists a compound state $\Phi$ satisfying

$$
I_{L}\left(\rho ; \Lambda_{0}^{*}\right)=S\left(\Phi, \rho \otimes \Lambda_{0}^{*} \rho\right)
$$

Theorem 2.11. For the attenuation channel $\Lambda_{0}^{*}$ and the input state

$$
\rho=\lambda|0\rangle\langle 0|+(1-\lambda)| \theta\rangle\langle\theta|,
$$


if $\lambda=\frac{1}{2}$ and $\alpha=1$, then there exists a compound state $\Phi$ satisfying

$$
S\left(\Phi, \rho \otimes \Lambda_{0}^{*} \rho\right)=S(\rho) .
$$

Acknowledgment. The author would like to thank Prof. I. Volovich for his many helpful sugestions during the preparation of the paper.

\section{REFERENCES}

1. M. Ohya, "Information dynamics and its application to optical communication processes" / In: Quantum Aspects of Optical Communications (Paris, 1990) / Lecture Notes in Physics, 378. Berlin: Springer, 1991. Pp. 81-92.

2. M. Ohya, "On compound state and mutual information in quantum information theory" // Information Theory, IEEE Trans., 1983. Vol. 29, no. 5. Pp. 770-774.

3. M. Ohya, D. Petz, Quantum entropy and its use/ Texts and Monographs in Physics. Springer Verlag: Berlin, 1993. viii+335 pp.

4. M. Ohya, I. Volovich, Mathematical foundations of quantum information and computation and its applications to nano- and bio-systems / Theoretical and Mathematical Physics. Dordrecht: Springer, 2011. xx+759 pp.

5. R. S. Ingarden, A. Kossakowski, M. Ohya, Information dynamics and open systems. Classical and quantum approach / Fundamental Theories of Physics. Vol. 86. Dordrecht: Kluwer Academic Publ., 1997. x+307 pp.

6. M. Ohya, N. Watanabe, "Construction and analysis of a mathematical model in quantum communication processes" // Electronics and Communications in Japan, Part 1, 1985. Vol. 68, no. 2. Pp. 29-34.

7. L. Accardi, M. Ohya, "Compound channels, transition expectations, and liftings" // Appl. Math. Optim., 1999. Vol. 39, no. 1. Pp. 33-59.

8. K.-H. Fichtner, W. Freudenberg, V. Liebscher, Beam splittings and time evolutions of Boson systems: Forschungsergebnise der Fakultät für Mathematik und Informatik, Math/Inf/96/39, 1996. 105 pp.

9. J. von Neumann, Die Mathematischen Grundlagen der Quantenmechanik (German). Berlin: Springer Verlag, 1932. v+262 pp.

10. M. Ohya, "Some aspects of quantum information theory and their applications to irreversible processes" // Rep. Math. Phys., 1989. Vol. 27, no.1. Pp. 19-47.

11. K. Urbanik, "Joint probability distribution of observables in quantum mechanics" // Studia Math., 1961. Vol. 21, no. 1. Pp. 117-133.

12. R. Schatten, Norm ideals of completely continuous operators / Ergebnisse der Mathematik und ihrer Grenzgebiete. Vol. 27. Berlin, New York: Springer Verlag, 1970. vii+81 pp.

13. H. Umegaki, "Conditional expectation in an operator algebra. IV. Entropy and information" // Kōdai Math. Sem. Rep., 1962. Vol. 14. Pp. 59-85.

14. H. Araki, "Relative entropy for states of von Neumann algebras" // Publ. Res. Inst. Math. Sci., 1976. Vol. 11, no. 3. Pp. 809-833.

15. B. W. Schumacher, M. A. Nielsen, "Quantum data processing and error correction" // Phys. Rev. A, 1996. Vol. 54, no. 4. Pp. 2629-2635, arXiv: quant-ph/9604022.

16. A. Uhlmann, "Relative entropy and the Wigner-Yanase-Dyson-Lieb concavity in interpolation theory" // Commun. Math. Phys., 1977. Vol.54, no. 1. Pp. 21-32.

17. M. Ohya, D. Petz, N. Watanabe, "On capacity of quantum channels" // Probab. Math. Statist., 1997. Vol. 17, no. 1 (Acta Univ. Wratislav. No. 1928). Pp. 179-196.

18. M. Ohya, D. Petz, N. Watanabe, "Numerical computation of quantum capacity", Proc. of the International Quantum Structures Association (Berlin, 1996)// Internat. J. Theoret. Phys., 1998. Vol. 37, no. 1. Pp. 507-510. 
19. M. Ohya, N. Watanabe, "Quantum capacity of noisy quantum channel" // Quantum Communication and Measurement, 1997. Vol. 3. Pp. 213-220.

20. M. Ohya, N. Watanabe, Foundation of Quantum Communication Theory (in Japanese): Makino Pub. Co., 1998.

21. P. Shor, The quantum channel capacity and coherent information: Lecture Notes, MSRI Workshop on Quantum Computation, 2002.

22. H. Barnum, M. A. Nielsen, B. W. Schumacher, "Information transmission through a noisy quantum channel" // Phys. Rev. A, 1998. Vol.57, no.6. Pp. 4153-4175, arXiv: quant-ph/9702049.

23. C. H. Bennett, P. W. Shor, J. A. Smolin, A. V. Thapliyalz, "Entanglement-assisted capacity of a quantum channel and the reverse Shannon theorem" // Inform. Theory, IEEE Trans., 2002. Vol. 48, no. 10. Pp. 2637-2655, arXiv: quant-ph/0106052.

24. B. W. Schumacher, "Sending entanglement through noisy quantum channels" // Phys. Rev. A, 1996. Vol. 54, no. 4. Pp. 2614-2628.

25. M. Ohya, N. Watanabe, Comparison of mutual entropy-type measures: TUS preprint.

Original article submitted 14/XI/2012; revision submitted 21/I/2013. 